\title{
TENSIONES EN LA ENSEÑANZA Y APRENDIZAJE DE LA HISTORIA EN CONTEXTO INTERÉTNICO: SIGNIFICACIONES Y EXPERIENCIAS ESCOLARES DE JÓVENES PEHUENCHES
}

\author{
TENSIONS IN TEACHING AND LEARNING HISTORY IN \\ AN INTERETHNIC CONTEXT: SIGNIFICANCE FOR AND \\ SCHOOL EXPERIENCES OF PEHUENCHE YOUNG STUDENTS
}

\section{OMAR TURRA DÍAZ**}

\section{RESUMEN}

El artículo presenta resultados de una investigación que tuvo como propósito indagar en el significado y valoración que respecto de la enseñanza de la historia posee el estudiantado pehuenche de la comuna de Alto Biobío, en el contexto de las nuevas finalidades de la enseñanza de la historia y los derechos educativos de los pueblos originarios. El estudio se realizó desde la metodología cualitativa con un diseño de estudio de casos, utilizándose como técnica de recogida de información el grupo de discusión. Los resultados evidencian aprendizajes relevantes asociados a la cultura de referencia, la presencia de conflictividad y discriminación en su enseñanza y una escasa valoración de la historia como disciplina escolar.

Palabras clave: Enseñanza de la historia, pueblos originarios, pehuenches, contexto interétnico.

\section{ABSTRACT}

This article presents results of a qualitative study whose purpose was to find out the meaning and value that Pehuenche students from the Alto Biobío commune give to the teaching of history in the context of the new goals of the teaching of history and the educational rights of the indigenous peoples. The study was conducted using a case study

* El artículo se enmarca en el proyecto Fondecyt Regular $N^{\circ} 1151233$ "La historia escolar en contextos interétnicos e interculturales: un estudio desde el sistema escolar en contexto mapuche/ no mapuche".

** Doctor en Educación, docente Facultad de Educación y Humanidades de la Universidad del Bío-Bío, Chile. Correo electrónico: oturra@ubiobio.cl 
design and the information was collected through the discussion group technique. The results show relevant learning associated with the reference culture, the presence of conflict and discrimination in their education and little appreciation of history as a school subject.

Keywords: teaching of history, indigenous peoples, Pehuenche, ethnic context.

Recibido: 17.01.14. Aceptado: 28.03.16.

\section{INTRODUCCIÓN}

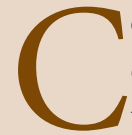

OMO POCAS ASIGNATURAS del currículo escolar, cada cierto tiempo la enseñanza de la historia es fuente de controversia acerca de su estatus en el sistema educativo y su rol formativo en la sociedad actual. Es quizás, su naturaleza ideológica, política y cultural -más expuesta que otras disciplinas escolares- la que mejor explica los debates acerca de sus finalidades educativas y la permanente "vigilancia" que ejercen los gobiernos sobre su enseñanza, cuestión que en determinados momentos históricos ha devenido en usos y abusos en su trayectoria como asignatura. Sin embargo, existe consenso acerca del rol clave que desempeña este sector de aprendizaje en la formación de los sujetos y en la construcción de las sociedades, dado su carácter de gestora de conciencia y de identidad.

Las actuales orientaciones educativas de su enseñanza plantean como una de sus finalidades el contribuir a la afirmación identitaria de los estudiantes (MINEDUC, 2009), es decir, los aprendizajes alcanzados en la asignatura servirían al estudiantado para reconocerse en su cultura y, desde ahí, conocer otras en una dimensión ascendente. En términos declarativos, este propósito se plantea en consecuencia con el reconocimiento de la diversidad cultural y los derechos educativos de los pueblos originarios en Chile, para quienes se ha oficializado el derecho a una formación en su cultura e historia a través del sistema educativo. ${ }^{1}$

A partir de las nuevas finalidades de la enseñanza de la historia y los derechos educativos indígenas, el artículo presenta los resultados de una in-

\footnotetext{
${ }^{1}$ En el caso de los pueblos originarios, los propósitos educativos orientados a una afirmación identitaria se corresponden con el reconocimiento estatal al derecho de mantener y desarrollar sus propias manifestaciones culturales (Ley 19253, art. 7, del año 1993) y con el reconocimiento de los derechos educativos de los pueblos indígenas a fin de responder a sus necesidades particulares, $y$ deberán abarcar su historia, sus conocimientos y técnicas (Convenio 169 OIT, art. 27, en vigencia desde el año 2009), aspectos que deben ser promovidos por los sistemas e instituciones educativas.
} 
vestigación que tuvo como propósito indagar en el significado y valoración que respecto de la enseñanza en esta asignatura escolar posee el estudiantado pehuenche de la comuna de Alto Biobío.

\section{MARCO TEÓRICO}

La historia como asignatura escolar se presenta como uno de los principales dispositivos simbólicos utilizados por los grupos de poder, para desplegar procesos formativos orientados a la construcción de identidades nacionales (Prats, 2000; Carretero y Castorina, 2010). Desde la época fundacional del estado y durante buena parte del siglo XX, el discurso pedagógico de la historia como disciplina escolar se asoció -se asocia- a un repertorio de imágenes heroicas que recreaban la trayectoria patria y a una representación del pasado social como constitutiva de una "comunidad cultural", que tenía como propósito explícito interiorizar en las cohortes estudiantiles el sentido de pertenencia a una nación y cultura común. La narrativa histórica gestionada ideológicamente por la cultura dominante buscaba unir las conciencias en torno a una causa superior que estaba por encima de cualquier grupo cultural, quedando éstos subsumidos en un marco estatal supracultural.

No obstante, la finalidad ideológico-nacionalista de la historia enseñada, además de su carácter enciclopédico y universalista, fue puesta en entredicho por estudios como el de Goodson² (1995), que analizaba el proceso mediante el cual determinados campos de conocimiento se convirtieron en disciplinas escolares, cuestión que llevó a la generación de discursos renovadores acerca del rol pedagógico y la importancia del saber histórico en el currículo educativo. Es así como Prats (2001) enfatiza la potencialidad formadora a nivel integral promovida por la enseñanza de la historia, en tanto contribuye al desarrollo intelectual, social y afectivo de los sujetos escolares. También se reconoce su aportación a la comprensión del presente, puesto que ofrece una perspectiva de análisis -centrado en los antecedentes- que infunde riqueza y relevancia al estudio de los fenómenos sociales contemporáneos (Merchán, 2002; Prat, 2001). Otros autores (Aranguren, 2005; Pagés, 2007) visualizan en su enseñanza una finalidad política, en

\footnotetext{
${ }^{2}$ Para este autor, el currículo es una construcción histórica y cultural en que intervienen diversas fuerzas. Es un producto socio-histórico, cruzado por múltiples intereses, relaciones y discursos que en definitiva son los que legitiman las asignaturas y los contenidos de enseñanza en el sistema escolar.
} 
cuanto instancia educativa orientada a la formación de ciudadanos, para una proyección democrática de las sociedades, y a la preservación de la memoria colectiva de los pueblos.

Desde el reconocimiento de la multiculturalidad de las sociedades y valoración de la diversidad cultural, se ha definido la enseñanza de la historia como un ámbito propicio para la conformación de subjetividades interculturales (Galindo, 2005; Valls, 2005), pues prepara al estudiantado para comprender(se) y reconocer(se) a(en) los otros culturales, aportando con ello a la tarea de eliminar males sociales como prejuicios, estereotipos, etnocentrismo y racismo. No obstante, para avanzar hacia estos propósitos socioeducativos se requeriría al menos dos transformaciones de orden pedagógico; una dirigida a la reestructuración de los programas de estudio que permitan multiplicar los enfoques o miradas culturales, para dar cabida a la dimensión plural y compleja de las sociedades; la otra, el ejercicio de un pensamiento histórico centrado en la diferencia y alteridad, situación que favorecería el desarrollo de virtudes como la descentración y la empatía, virtudes que se constituyen en protección ante el etnocentrismo (Galindo, 2005).

En los sentidos de los nuevos discursos respecto de la finalidad de la enseñanza de la historia, se evidencia el propósito común de superar el relato tradicional lineal y monocultural, funcional a la conformación de las identidades nacionales, promoviéndose, por el contrario, una orientación pedagógica problematizadora de los contenidos históricos y de la realidad social, cuestión que involucra construir una teoría y práctica formativa centrada en la complejidad sociocultural, donde se expresen diferentes universos culturales e identitarios.

Estos nuevos enfoques en la enseñanza de la historia se encuentran en concordancia con aquellas propuestas pedagógicas que promueven la pertinencia cultural de los procesos formativos (Torres, 2008; Pinto, 2008); la construcción de identidad como objetivo educativo, en tanto se programa la enseñanza a partir de las experiencias, saberes previos y visiones de mundo que porta el estudiantado (OREAL/UNESCO, 2008); y con las orientaciones propias del sector de aprendizaje que busca desarrollar "una actitud de respeto a la diversidad cultural de la humanidad y del país, junto con un sentido de pertenencia" (MINEDUC, 2009, p. 195). En esta perspectiva, la enseñanza de la historia no solo adquiere un rol de privilegio en la conformación de subjetividades interculturales, sino que también contribuye a la generación de igualdad educativa al incorporar categorías, saberes y lenguajes que portan los grupos estudiantiles pertenecientes a culturas minoritarias o minorizadas, como ocurre con el estudiantado que proviene 
de los pueblos originarios y que se desarrolla social y educativamente en el contexto de relaciones interétnicas.

Sin embargo, estudios empíricos realizados desde el ámbito cultural mapuche plantean la desconsideración del conocimiento indígena en los procesos formativos que se desarrollan en las escuelas ubicadas en este contexto (Quilaqueo, Quintriqueo y Cárdenas, 2005) y la prevalencia de un currículo monocultural (Quintriqueo, 2010). Respecto de las actuaciones docentes, se ha evidenciado que el profesorado en sus procesos pedagógicos desarrolla una serie de prácticas poco proclives a la consideración de lo indígena y, más aún, se desarrollan prejuicios y sesgos hacia sus prácticas culturales (Quilaqueo y Merino, 2003; UNESCO, 2005).

Desde la perspectiva de la enseñanza de la historia, otra investigación que analiza los programas de estudio de la asignatura constata el reducido espacio curricular para la enseñanza de las formas culturales mapuche y la ausencia de una narrativa histórica que otorgue espacio a las visiones de los pueblos originarios, es decir su estudio se realiza desde la perspectiva del "otro" (Turra, 2012). Un estudio centrado en las significaciones docentes (Turra, 2015) expone que el profesorado coincide en atribuir una alta relevancia al conocimiento cultural mapuche como saber escolar, pero al mismo tiempo los educadores manifiestan el escaso conocimiento que poseen de los saberes histórico-educativos mapuche, además de la ausencia de un trabajo pedagógico con estos saberes en la enseñanza de la historia. Se concluye que, no obstante los discursos de reconocimiento y valoración de la diversidad cultural, de los derechos educativos y culturales indígenas, la historia escolar en contexto interétnico propone y promueve unos conocimientos culturales eurocéntricos que reproducen el tradicional discurso pedagógico de la historia.

\section{METODOLOGÍA}

El estudio se realizó desde la metodología cualitativa de investigación, dada su intencionalidad de búsqueda de la comprensión de una realidad social determinada, aportada por las significaciones de los propios sujetos que participan del campo objeto de investigación (Rodríguez et al., 1999).

El enfoque utilizado es el fenomenológico, puesto que se busca construir conocimiento a partir de la experiencia subjetiva de los sujetos, describiendo e interpretando los significados comunes aportados por los sujetos de investigación. 
En términos del diseño, se trabajó con la modalidad estudio de caso. Este tipo de estudio permite investigar con cierta intensidad y profundidad un fenómeno social, una institución, un grupo social u otro. En la investigación, el caso lo constituye el estudiantado pehuenche, que desarrolla su escolaridad en el Liceo Técnico Intercultural Bilingüe de Ralco, comuna de Alto Biobío y que representa, históricamente, el $85 \%$ de la matrícula de esta institución educativa.

De acuerdo con los objetivos de la investigación, el caso se construyó a partir de los siguientes criterios: estudiantes de ascendencia mapuchepehuenche, que se encuentren cursando $2^{\circ}$ y/o $3^{\circ}$ medio, y voluntariedad en su participación en el estudio. Así el caso quedó compuesto por ocho escolares que se caracterizan en el siguiente cuadro:

Caracterización general del caso

\begin{tabular}{|c|c|c|}
\hline Sujeto & Género & Nivel \\
\hline estudiante 1 & femenino & $3^{\circ}$ medio \\
\hline estudiante 2 & masculino & $2^{\circ}$ medio \\
\hline estudiante 3 & masculino & $3^{\circ}$ medio \\
\hline estudiante 4 & masculino & $3^{\circ}$ medio \\
\hline estudiante 5 & masculino & $3^{\circ}$ medio \\
\hline estudiante 6 & femenino & $2^{\circ}$ medio \\
\hline estudiante 7 & femenino & $2^{\circ}$ medio \\
\hline estudiante 8 & femenino & $2^{\circ}$ medio \\
\hline
\end{tabular}

La técnica de recogida de información que se utilizó fue el grupo de discusión, en tanto estrategia que permite confrontar la subjetividad individual con la grupal, y pretende poner en contacto diferentes perspectivas, experiencias y puntos de vista (Valles, 2007). En tres momentos distintos se produjo encuentro entre el investigador y los participantes del caso, para alcanzar los propósitos del estudio.

La reducción de la información se realizó de acuerdo al método fenomenológico, codificándose los registros comunicativos que expresan sentidos compartidos o de consenso, es decir la esencia del significado que el estudiantado comparte intersubjetivamente respecto del fenómeno investigado. La concentración de significados comunes expresan las categorías o unidades de análisis y de ellas se presentan los relatos más representativos como sus resultados. 


\section{RESULTADOS}

Los resultados que surgieron del grupo de discusión se pueden sintetizar en la siguiente tabla:

\begin{tabular}{|l|l|c|}
\hline \multicolumn{1}{|c|}{ Categoría } & Concentración de significado & $\begin{array}{c}\text { Registros } \\
\text { comunicativos }\end{array}$ \\
\hline $\begin{array}{l}\text { Aprendizajes relevantes en la } \\
\text { asignatura }\end{array}$ & $\begin{array}{l}\text { Colonización, mestizaje, } \\
\text { defensa del territorio }\end{array}$ & 4 \\
\hline $\begin{array}{l}\text { Distancia entre historia enseñada } \\
\text { y cultura de referencia }\end{array}$ & $\begin{array}{l}\text { Descontextualización, } \\
\text { valoración de relatos de los } \\
\text { abuelos }\end{array}$ & 3 \\
\hline $\begin{array}{l}\text { Discriminación en la clase de } \\
\text { historia }\end{array}$ & $\begin{array}{l}\text { Presencia de estereotipos, } \\
\text { cuestionamiento al concepto } \\
\text { de indígena }\end{array}$ & 3 \\
\hline Valoración de la clase de historia & Escasa valoración & 3 \\
\hline
\end{tabular}

\section{Aprendizajes relevantes en la asignatura}

La concentración de significado se produjo aquí en torno a la identificación de aprendizajes relevantes con procesos históricos vinculados a su cultura de referencia -pehuenche- o más bien con aquellos procesos sociales que vinieron a pauperizar las culturas originarias. En este sentido, los aprendizajes enunciados como relevantes por los y las estudiantes se pueden definir en las siguientes temáticas: la colonización española-chilena; el mestizaje; también aquellos contenidos asociados a la defensa del territorio ancestral por los pueblos originarios.

A mí me llama la atención la colonización. Sí, la colonización chilena... en estos territorios. Porque hubieron mucho, este, por ejemplo los españoles se aprovechaban mucho de la gente indígena, igual hubieron hartas muertes, igual por inequidad... Me gustó un poco porque nosotros defendimos nuestro territorio, pero igual no me gustó porque se aprovechaban mucho de las personas (Estudiante 4).

Por mi parte fue cuando Chile hizo una mezcla de todo tipo de personas, entre los blancos y entre los indígenas, y a mí me gustó demasiado porque vivo en un país que está mestizado... que yo me acuerde no sé cómo se llamaba cierta persona, por ejemplo la gente zamba, era la gente indígena con negro, entonces yo no sabía que se llamaba así (Estudiante 1). 
Los relatos del estudiantado se encuentran en concordancia con los principios del diseño curricular vigente, que postula el aprendizaje significativo de los/las aprendices y este comienza con el rescate de las ideas y experiencias previas. Además un aprendizaje será relevante en la medida en que se vincule con las realidades propias del entorno social y cultural en que se desenvuelve el estudiantado. Al parecer en la clase de historia no se realizan las debidas vinculaciones temáticas con la realidad del estudiantado mapuche-pehuenche.

hacen otros temas pero igual cuando pasan esa materia es interesante (Estudiante 3).

... historia mapuche no vemos mucho, sólo en la básica, en la media no (Estudiante 4)

La categorización de los aprendizajes relevantes relevados por el estudiantado indígena lleva implícita la demanda por una enseñanza de la historia vinculada a la realidad contextual.

\section{Distancia entre historia enseñada y cultura de referencia}

Esta categoría surge de la indagación en las significaciones del estudiantado mapuche-pehuenche respecto de la vinculación del saber histórico escolar con su cultura de referencia. Las significaciones en este sentido se concentran, por una parte, en un diagnóstico negativo respecto de los saberes y temas que se enseñan en la asignatura:

Nosotros ahora estamos pasando eso que se llama feudalización, feudalismo, y los viajes de Colón, pero todo es como qué países conquistaron, nada de acá, sólo nos pasaron eso (Estudiante 1).

Por otra, se demanda por una clase más vinculada a la realidad contextual o cultura de referencia del estudiantado:

Yo creo que del $100 \%$ se ve el $45 \%$... Uno viene de la cultura mapuche, uno tiene ceremonias que se hacen, se siguen haciendo, nosotros queremos eso, estamos conservando y dependiendo más de ello (Estudiante 2). 
El estudiantado expone como alternativa a un conocimiento histórico descontextualizado, el diálogo con los abuelos, es decir la recurrencia a la tradición oral, fuente legítima de transmisión de conocimiento en las culturas originarias:

... de repente le pregunto a mis abuelos por qué la gente vive tan aislada, no vive en la ciudad, y me dicen que hay un problema con la gente, con los españoles y todo eso (Estudiante 1).

... no nos hablaban de historia; los abuelitos nomás, es interesante lo que cuentan ellos, porque está más relacionado con la vida que lo otro (Estudiante 5).

Lo interesante de estas observaciones, realizadas por los estudiantes, es que los abuelos son considerados aún por ellos como fuentes de conocimiento válido para adquirir aprendizajes respecto del pasado históricocultural, pero a la vez muestra la ineficacia del sistema escolar chileno (sus conformaciones y agentes educadores) para impactar significativamente en los aprendices pertenecientes a estas conformaciones culturales.

Sin embargo, al discutir la posibilidad de incorporar temas vinculados a su realidad cultural en la clase, muchos de ellos estuvieron en desacuerdo con aquello, pues sería una fuente de conflictos con los escasos compañeros hijos de colonos chilenos con los que comparten clases (15\%).

Porque cuando hablan de temas entre mapuche y españoles, yo veo a mi compañero del lado que no es mapuche, entonces me siento como... porque me siento con él me entra un rencor. A mí me pasó eso cuando estaba en primero, y me juntaba con compañeros que no eran mapuche, entonces cuando hablaban de esos temas, me sentía, así como enojada con sus descendientes (Estudiante 2)

Yo estoy de acuerdo con las respuestas de mis compañeros porque tenemos muchos conflictos con los compañeros y profesores también porque a veces hay una mala comprensión de ciertas cosas (Estudiante 4).

Lo interesante de las observaciones manifestadas por el estudiantado mapuche-pehuenche es que a pesar de ser ellos mayoría en el Liceo y en la clase, perciben que los temas relacionados con su cultura son fuente de discusión y conflicto para el profesorado y el escaso número de compañeros/ 
as chilenos/as que componen sus cursos; prefieren, por lo mismo, no agregar otros temas a la clase de historia que pudiesen profundizar estos conflictos.

\section{Discriminación en la clase de historia}

Una categoría que emergió de la discusión grupal de manera transversal a las preguntas planteadas, sin asociación explícita con ninguno de los objetivos planteados, fue aquella vinculada a declaraciones de discriminación en la clase de historia. Los y las estudiantes mapuche-pehuenches manifestaron que en la clase existían expresiones y acciones del profesorado y de sus compañeros/as chilenos/as que ofenden o al menos les generaba desagrado:

Una vez teníamos un profesor que escribió en la pizarra, los pehuenche son unos ignorantes, borrachos y otras cosas más; entonces uno siente rabia (Estudiante 4).

Hay un tema de discriminación, porque para hablar de los temas, se tiene que ver más la cosa, yo soy hablante de chedungun, hay compañeros (chilenos) que si uno habla se empiezan a llevar mal, yo como indígena esas cosas que pasan en historia, como por ejemplo, ya mataron a un indígena, a mí me empieza a dar rabia una cosa así, como que las cosas, la clase de historia, sí, son más que nada interesantes, son buenas, pero creo que hay muchas contradicciones entre chilenos y nosotros, la clase de historia para mí sería mejor, yo la dejaría de lado, porque empiezan como a discriminarse y como ahora estamos todos juntos comienzan a generarse conflictos, porque siguen recordando siempre lo mismo, por eso... (Estudiante 2).

Muchas veces la enseñanza de la historia por sus problemáticas es fuente de conflictos y discusiones, también es deseable que generen conflicto en el estudiantado -sobre todo si es de carácter cognitivo-, pero obviamente sin que ello genere afectaciones o discriminaciones que afecten a individuos o a grupos dentro de la clase.

A mí no me gusta hablar mucho de este tema porque a mí mismo me da mucha rabia que me digan indígena, prefiero que me digan mapuche o pehuenche, pero indígena, lo escuchamos mucho en la clase y uno tiene que guardarse la opinión (Estudiante 3 ).

Esta demanda de una estudiante no es menor, considerando la impronta o carga histórica negativa que lleva el concepto de indígena, y que 
las declaraciones curriculares e incluso las legales (Ley Indígena) la incorporan como lenguaje aceptado, pero que a los sujetos alejados/as de estos centros de configuración conceptual les incomoda o definitivamente las rechazan.

\section{Valoración de la clase de historia}

La concentración de sentido se produce en torno a una escasa valoración por parte del estudiantado respecto de la clase de historia, según se desprende de sus relatos, en tanto se presenta la asignatura como una instancia donde se estudian temáticas que ocurren en tiempos remotos, vinculados a otras culturas y otros espacios geográficos, y las problemáticas relacionadas con las realidades culturales contextuales no son parte de la clase:

Porque a nosotros nos enseñan historia universal y de repente no entendemos nada, eso que pasó allá, nosotros queremos saber que pasó acá, incluso nuestra tierra... de acá sigue haciendo...pasó hace algunos años y a mí me gustaría saberlos, todos los años va haciendo historia (Estudiante 5).

Es que en la clase, por mi parte no tocamos ese tema, no hablamos de los mapuche, hablamos de otro tema, de ¿cómo se llama?... feudalismo, $\mathrm{y}$ todas esas cosas (Estudiante 2).

Cuando se les pregunta respecto del conjunto de asignaturas que cursan o han cursado, en qué lugar situarían la clase de historia en términos de su interés, la respuesta la ubica entre las menos atractivas.

las asignaturas que más nos gustan son Biología, inglés... (varias voces).

En rigor, la escasa valoración que aduce el estudiantado mapuchepehuenche no estaría lejos de lo que pudiese manifestar un/a estudiante chileno/a que asiste a las clases de historia de un liceo chileno, la diferencia es que la vinculación con aspectos de la cultura europea para unos adquiere sentidos de pertinencia curricular (estudiantado chileno) y para el otro definitivamente deviene en configuraciones culturales que habría que referenciar (estudiante indígena), pero no ser el centro del estudio en la asignatura, descuidando lo propio como saber legítimo en el sistema escolar. 


\section{DISCUSIÓN}

De acuerdo con los hallazgos investigativos, resulta evidente que los nuevos propósitos de la enseñanza de la historia no se han manifestado en la experiencia educativa de los jóvenes escolares pehuenches, en particular aquel vinculado a la construcción de subjetividades interculturales a partir del reconocimiento de la propia cultura. Tampoco se visualiza la implementación de procesos pedagógicos que promuevan la afirmación identitaria de este conglomerado estudiantil de acuerdo con las declaraciones curriculares en la asignatura, presentándose más bien un cuerpo de contenidos asociados a la tradición europea occidental y a la trayectoria de la sociedad colonial/criolla/chilena en su conformación como organización estatal.

Considerando lo anterior, la cultura originaria pehuenche que está a la base de la gran mayoría del estudiantado que asiste a este centro educacional, no se visualiza como una fuente de sentidos social e histórico, como una sociedad con trayectoria soberana y de relaciones interétnicas, respecto de la corona española y el Estado chileno. Los relatos del estudiantado dan cuenta del interés por temáticas cercanas a su realidad cultural y que, de manera positiva o negativa, dicen algo respecto de las sociedades originarias, pero en todo caso la narrativa histórica se realiza desde una perspectiva nacional y eurocéntrica. Al desconsiderar la enseñanza de la historia, el estudio y conocimiento de la trayectoria sociocultural pehuenche, los relatos de los abuelos se constituye en una instancia de sobrevivencia cultural, en la medida en que organiza la memoria social para proyectarla en la formación de las nuevas generaciones.

Por el contrario, desde la perspectiva de la enseñanza de la historia se imposibilita el ejercicio de un pensamiento histórico que construya y favorezca la diferencia y la alteridad en el alumnado, cuestiones fundamentales si se postula la afirmación identitaria y la construcción de subjetividades interculturales como finalidades de su enseñanza. Desde esta comprensión, la historia enseñada en el sistema escolar se configura como una expresión de la colonialidad del poder y el saber, en tanto práctica discursiva que incuba violencia epistémica (Castro Gómez, 2000) cuando asigna superioridad a unos conocimientos derivados del mundo europeo y por el contrario excluye, omite y silencia los conocimientos de las culturas originarias, produciendo una subalternización de estos pueblos y su lenguaje.

Las características socioculturales del alumnado participante del estudio, su pertenencia a un pueblo originario que conserva pautas culturales tradicionales en la organización de su existencia, su situación sociolingüís- 
tica que hace de ellos/as, en su mayoría hablantes de la lengua materna -chedungun-, expresan una identidad cultural de base que contribuye a explicar las situaciones de conflictividad generadas en la enseñanza de la asignatura y la discriminación percibida en las relaciones pedagógicas.

Por otra parte, estas expresiones de conflictividad y discriminación que exponen los estudiantes refieren a fenómenos propios de sociedades en contacto de relaciones interétnicas, en los que se manifiestan situaciones de dominación y control simbólico. En este caso, la mayoría estudiantil pehuenche (85\%) es minorizada culturalmente en el centro escolar, al desplegarse unos objetivos y finalidades educativas que se corresponden con dimensiones propias de la sociedad y cultura dominantes, que en su mejor expresión representa al 15\% del estudiantado no pehuenche que asiste al Liceo de Ralco.

Los resultados del estudio vienen a reafirmar aquellos hallazgos investigativos que constatan, para contextos específicos de indigenidad mapuche, la exclusión de los saberes culturales originarios en los programas de estudio (Quilaqueo et al., 2005; Quintriqueo, 2010) y la existencia de situaciones de prejuicios y discriminación hacia las formas culturales indígenas por parte del profesorado (Quilaqueo y Merino, 2003; UNESCO, 2005). También se constata la ausencia de una narrativa histórica que otorgue espacio a las visiones de los pueblos originarios, promoviéndose una historia escolar monocultural en que el pueblo mapuche es el "otro" inventado y su presencia como protagonista en este relato se expresa en la relación/oposición al proyecto histórico hispano-chileno.

El profesorado, protagonista secundario en este estudio, es sin duda fundamental en los procesos de significación y valoración de la enseñanza de la historia que realiza el estudiantado, y sus actuaciones en el contexto de estudio llevan a sospechar que, cuando el discurso educativo demanda nuevas prácticas de enseñanza para responder a los desafíos de la interculturalidad, se le impone un requerimiento de competencias profesionales para el cual no fue, necesariamente, habilitado en la formación inicial.

Por otra parte, el ámbito de los fines e intencionalidad educativa se produce y desarrolla en planos que no siempre se vincula y/o correlaciona con las actuaciones pedagógicas, con los propósitos que movilizan las actuaciones docentes en el sistema escolar, pero sin duda que es el profesorado el que en último término definirá el sentido y profundidad de los nuevos derroteros de la enseñanza de la historia en los contextos específicos de enseñanza. Como plantea Besalú son los profesores los que 
con sus palabras y sus conductas, con sus miradas y sus gestos, con sus creencias y prejuicios, promueven y estimulan el aprendizaje, quienes pueden legitimar la prevalencia de la cultura hegemónica, profundamente discriminadora y sesgada o quienes pueden dar carta de naturaleza a una cultura más amplia y más representativa, más funcional y más justa (2004, p. 56).

\section{CONCLUSIONES}

La enseñanza de la historia en su dimensión pedagógica, si bien ha renovado sus finalidades educativas para ajustarse a las demandas y complejidades socioeducativas contemporáneas, se identifica aún con unas prácticas escolares asociadas a los sentidos tradicionales de la enseñanza en la asignatura. Es decir, se visualiza un desajuste entre la intencionalidad educativa y las prácticas de formación desarrolladas en la escuela.

En un contexto escolar interétnico, como el estudiado, atravesado por relaciones sociales y culturales asimétricas, la enseñanza de la historia se representa como un dispositivo cultural privilegiado de la colonialidad del poder y del saber, en tanto valida unos contenidos escolares y una perspectiva de estudio monocultural que se vincula directamente con la tradición cultural europea. Como contrapartida, subalterniza los conocimientos y cosmovisiones de los pueblos originarios, así como su propia trayectoria histórica.

Las significaciones aportadas por los estudiantes pehuenches respecto de la historia escolar -aprendizajes relevantes vinculados a la cultura de referencia, conflictividad y discriminación en el aula- evidencian la lógica tradicional de su enseñanza y tal vez sea ella, también, la razón explicativa de su escasa valoración.

\section{REFERENCIAS}

Aranguren, C. (2005). "Teoría y Praxis en la enseñanza de la historia: una relación epistemológica”. Educere, 9(28), 61-65.

Besalú, X. (2004). “La formación inicial en interculturalidad”. En Jordán, J. et al. (Eds.), La formación del profesorado en Educación Intercultural (pp. 4992). Madrid: Ministerio de Educación, Cultura y Deporte.

Carretero, M. y Castorina, J. (2010). La construcción del conocimiento histórico. Enseñanza, narración e identidades. Buenos Aires: Paidós.

Castro Gómez, S. (2000) "Ciencias sociales, violencia epistémica y el proble- 
ma de la 'invención' del otro”. En: Lander, E. (Comp.), La colonialidad del saber: eurocentrismo y ciencias sociales. Perspectivas latinoamericanas (pp. 145-161). Buenos Aires: CLACSO.

Galindo, R. (2005). "Cambios curriculares en Ciencias Sociales para responder a la multiculturalidad”. En García, C. et al. (Eds.), Enseñar Ciencias Sociales en una sociedad multicultural: Una mirada desde el Mediterráneo (pp. 305343). Almería: Asociación Universitaria del profesorado de Didáctica de las Ciencias Sociales.

Goodson, I. (1995). Historia del Curriculum. La construcción social de las disciplinas escolares. Barcelona: Pomares.

Merchán, F. (2002). "Profesores y alumnos en la clase de historia". Cuadernos de Pedagogía, 309, 90-94.

MINEDUC (2009). Objetivos Fundamentales y Contenidos Mínimos Obligatorios de la Educación Básica y Media. Santiago: Unidad de Currículum y Evaluación.

OREAL/UNESCO (2008). Educación y diversidad cultural. Lecciones desde la práctica innovadora en América Latina. Santiago: Pehuén.

Pagés, J. (2007). “La educación para la ciudadanía y la enseñanza de la historia: cuando el futuro es la finalidad en la enseñanza del pasado”. En Ávila, M. et al. (Eds.). Las competencias profesionales para la enseñanza-aprendizaje de las Ciencias Sociales ante el reto europeo y la Globalización (pp. 205-215). Bilbao: Asociación Universitaria del Profesorado de Didáctica de las Ciencias Sociales.

Pinto, R. (2008). El currículo crítico. Una pedagogía transformativa para la educación latinoamericana. Santiago: Ediciones PUC.

Prats, J. (2000). "Dificultades para la enseñanza de la historia en la Educación Secundaria: reflexiones ante la situación española”. Revista de Teoría y Didáctica de las Ciencias Sociales, 5, 71-98.

Prats, J. (2001). Enseñar Historia: Notas para una didáctica renovadora. Mérida: Junta de Extremadura. Consejería de Educación, Ciencia y Tecnología.

Quilaqueo, D. y Merino, M. (2003). "Estereotipos y prejuicio étnico hacia los mapuches en textos complementarios a la asignatura de Historia”. Campo Abierto, 23, 119-135.

Quilaqueo, D.; Quintriqueo, S. y Cárdenas, P. (2005). Educación, Currículum e Interculturalidad. Elementos sobre formación de profesores en contexto mapuche. Temuco: Universidad Católica de Temuco.

Quintriqueo, S. (2010). Implicancias de un modelo curricular monocultural en contexto mapuche. Temuco: Ediciones Universidad Católica de Temuco.

Rodríguez, J.; Gil, J. y García, E. (1999). Metodología de la investigación cualitativa. Málaga: Aljibe.

Torres, J. (2008). Diversidad cultural y contenidos escolares. Revista de Educación, 345, 83-110.

Turra, O. (2012). "Currículo y construcción de identidad en contextos indígenas chilenos". Educación y Educadores, 15(1), 81-95. 
Turra, O. (2015). "Profesorado y saberes histórico-educativos mapuche en la enseñanza de historia”. Revista Electrónica Educare, 9(3), 1-20.

UNESCO (2005). La discriminación y el pluralismo cultural en la Escuela. Casos de Brasil, Chile, Colombia, México y Perú. Santiago: OREAL/UNESCO.

Valles, M. (2007). Técnicas cualitativas de investigación. Madrid: Editorial Síntesis.

Valls, R. (2005). "La multiculturalidad en la enseñanza de la historia y de las ciencias sociales: el desajuste entre intenciones educativas y prácticas escolares y los retos del futuro educativo inmediato”. En García, C. et al. (Eds.), Enseñar Ciencias Sociales en una sociedad multicultural: Una mirada desde el Mediterráneo (pp. 263-277). Almería: Asociación Universitaria del profesorado de Didáctica de las Ciencias Sociales. 